\title{
The visual core of a hyperbolic 3-manifold
}

\author{
James W. Anderson and Richard D. Canary* \\ Faculty of Mathematical Studies, University of Southampton \\ Highfield, Southampton, SO17 1BJ, England \\ Department of Mathematics, University of Michigan, Ann Arbor, MI 48109
}

\begin{abstract}
In this note we introduce the notion of the visual core of a hyperbolic 3-manifold $N=\mathbf{H}^{3} / \Gamma$, and explore some of its basic properties. We investigate circumstances under which the visual core $\mathcal{V}\left(N^{\prime}\right)$ of a cover $N^{\prime}=\mathbf{H}^{3} / \Gamma^{\prime}$ of $N$ embeds in $N$, via the usual covering map $\pi: N^{\prime} \rightarrow N$. We go on to show that if the algebraic limit of a sequence of isomorphic Kleinian groups is a generalized web group, then the visual core of the algebraic limit manifold embeds in the geometric limit manifold. Finally, we discuss the relationship between the visual core and Klein-Maskit combination along component subgroups.
\end{abstract}

\section{Introduction}

In this note we introduce the notion of the visual core of a hyperbolic 3-manifold $N=$ $\mathbf{H}^{3} / \Gamma$. One may think of the visual core as a harmonic analysis analogue of the convex core. Explicitly, the visual core $\mathcal{V}(N)$ is the projection to $N$ of all the points in $\mathbf{H}^{3}$ at which no component of the domain of discontinuity of $\Gamma$ has visual (equivalently harmonic) measure greater than half that of the entire sphere at infinity.

We investigate circumstances under which the visual core $\mathcal{V}\left(N^{\prime}\right)$ of a cover $N^{\prime}=\mathbf{H}^{3} / \Gamma^{\prime}$ of $N$ embeds in $N$, via the usual covering map $\pi: N^{\prime} \rightarrow N$. We begin by showing that the interior of $\mathcal{V}\left(N^{\prime}\right)$ embeds in $N$ when $\Gamma^{\prime}$ is a precisely QF-embedded subgroup of $\Gamma$, while

${ }^{*}$ Research supported in part by grants from the National Science Foundation 
$\mathcal{V}\left(N^{\prime}\right)$ itself embeds when $\Gamma^{\prime}$ is a nicely QF-embedded subgroup. We define the notions of precisely and nicely QF-embedded subgroups of a Kleinian group and prove these embedding theorems in Section 3.

Applying the results from [3], we are able to conclude that if the algebraic limit of a sequence of isomorphic Kleinian groups is a generalized web group, then the visual core of the algebraic limit manifold embeds in the geometric limit manifold. This result is part of our ongoing investigation of the relationship between algebraic and geometric limits of sequences of isomorphic Kleinian groups. To set notation, let $\mathcal{D}(G)$ denote the space of all discrete, faithful representations of $G$ into $\mathrm{PSL}_{2}(\mathbf{C})$. This space and the notions of algebraic and geometric convergence of Kleinian groups are discussed in more detail in Section 4.

Theorem 4.2: $\quad$ Let $G$ be a finitely generated, torsion-free, non-abelian group, let $\left\{\rho_{j}\right\} \subset$ $\mathcal{D}(G)$ be a sequence converging algebraically to $\rho \in \mathcal{D}(G)$, and suppose that $\left\{\rho_{j}(G)\right\}$ converges geometrically to $\widehat{\Gamma}$. If $\rho(G)$ is a generalized web group, then the visual core of $N=\mathbf{H}^{3} / \rho(G)$ embeds in $\widehat{N}=\mathbf{H}^{3} / \widehat{\Gamma}$ under the covering map $\pi: N \rightarrow \widehat{N}$.

There are two ways to view Theorem 4.2. On the one hand, one may think of it as a geometric analogue of the main result from [3], which asserts that under the same hypotheses, there is a compact core for the algebraic limit manifold which embeds in the geometric limit manifold. On the other hand, Theorem 4.2 can be thought of as a generalization of the result, proven in [4], that when the algebraic limit is a maximal cusp, the convex core of the algebraic limit manifold embeds in the geometric limit manifold. In fact, when $\Gamma$ is a maximal cusp, the visual and convex cores of $\mathbf{H}^{3} / \Gamma$ coincide.

In Section 5, we discuss the relationship between the visual core and Klein-Maskit combination along component subgroups. Klein-Maskit combination gives a geometric realization of the topological operation of gluing hyperbolizable 3-manifolds together along incompressible surfaces in their boundaries. While the topology underlying Klein-Maskit combination is well-understood, the geometry is more mysterious. For example, the convex core of a summand of a Klein-Maskit combination need not embed in the resulting manifold. However, we show in Theorem 5.6 that the (interior of the) visual core of a summand does embed in the resulting manifold.

There is a relationship between these two investigations, of limits of sequences of Kleinian groups and of Klein-Maskit combination, since in the case that the algebraic limit is a generalized web group, it is shown in [3] that the algebraic limit is a summand of a KleinMaskit decomposition of the geometric limit. 
This paper was completed while the first author was visiting Rice University, and he would like to thank the department there for their hospitality.

\section{The visual core}

Before describing the basic properties of the visual core, we give some definitions. A Kleinian group is a discrete subgroup of $\mathrm{PSL}_{2}(\mathbf{C})$, which we view as acting either on hyperbolic 3space $\mathbf{H}^{3}$ as isometries or on the Riemann sphere $\widehat{\mathbf{C}}$ as Möbius transformations. The action of $\Gamma$ partitions $\widehat{\mathbf{C}}$ into the domain of discontinuity $\Omega(\Gamma)$, which is the largest open subset of $\widehat{\mathbf{C}}$ on which $\Gamma$ acts properly discontinuously, and its complement the limit set $\Lambda(\Gamma)$. The stabilizer

$$
\operatorname{st}_{\Gamma}(\Delta)=\{\gamma \in \Gamma \mid \gamma(\Delta)=\Delta\}
$$

of a connected component $\Delta$ of $\Omega(\Gamma)$ is called a component subgroup of $\Gamma$.

Given a measurable set $X \subset \widehat{\mathbf{C}}$, consider the harmonic function $h_{X}$ on $\mathbf{H}^{3}$ defined by setting $h_{X}(y)$ to be the proportion of the geodesic rays emanating from $y \in \mathbf{H}^{3}$ which end in $X$. Though we do not explicitly use this formulation, analytically we can write $h_{X}$ in the ball model of hyperbolic 3 -space as

$$
h_{X}(y)=\frac{1}{4 \pi} \int_{X}\left(\frac{1-|y|^{2}}{|y-\zeta|^{2}}\right)^{2} d m(\zeta) .
$$

The visual hull of a Kleinian group $\Gamma$ is then defined to be

$$
\widetilde{\mathcal{V}}(\Gamma)=\left\{y \in \mathbf{H}^{3} \mid h_{\Delta}(y) \leq \frac{1}{2} \text { for all components } \Delta \text { of } \Omega(\Gamma)\right\} .
$$

The visual core $\mathcal{V}(N)$ of $N=\mathbf{H}^{3} / \Gamma$ is the quotient $\widetilde{\mathcal{V}}(\Gamma) / \Gamma$. Although $\mathcal{V}(N)$ is a closed subset of $N$, there is no reason, in general, to suppose that $\mathcal{V}(N)$ is a submanifold (or suborbifold) of $N$.

Our first observation is that the visual core of a hyperbolic 3-manifold with finitely generated fundamental group is non-empty unless its domain of discontinuity is connected and non-empty.

Proposition 2.1 Let $\Gamma$ be a finitely generated Kleinian group. Then $\tilde{\mathcal{V}}(\Gamma)$ is empty if and only if $\Omega(\Gamma)$ is connected and non-empty. 
Proof of Proposition 2.1: By definition, $\widetilde{\mathcal{V}}(\Gamma)=\mathbf{H}^{3}$ if and only if the domain of discontinuity $\Omega(\Gamma)$ of $\Gamma$ is empty.

If $\Omega(\Gamma)$ is connected and non-empty, then $\Gamma$ is a function group, which is a finitely generated Kleinian group whose domain of discontinuity contains a component invariant under the action of the group. Soma [12] shows that $\Gamma$ is then topologically tame, that is the orbifold $\mathbf{H}^{3} / \Gamma$ has a finite degree manifold cover which is homeomorphic to the interior of a compact 3-manifold. Corollary 1 from [6] then implies that $\Lambda(\Gamma)$ has measure zero, so that $h_{\Omega(\Gamma)}(x)=1$ for all $x \in \mathbf{H}^{3}$. In particular, we have that $\tilde{\mathcal{V}}(\Gamma)$ is empty.

If $\Omega(\Gamma)$ contains at least two components, let $\ell$ be a geodesic in $\mathbf{H}^{3}$ whose endpoints at infinity lie in distinct components $\Delta_{1}$ and $\Delta_{2}$ of $\Omega(\Gamma)$. The function $h_{\Delta_{1}}$ varies continuously between 0 and 1 on $\ell$, and so there exists a point $x$ on $\ell$ such that $h_{\Delta_{1}}(x)=\frac{1}{2}$. It follows that $x \in \tilde{\mathcal{V}}(\Gamma)$, and so $\tilde{\mathcal{V}}(\Gamma)$ is non-empty.

Proposition 2.1

It is natural to contrast the definition of the visual core with that of the convex core. Recall that the convex hull $\widetilde{\mathcal{C}}(\Gamma)$ of $\Lambda(\Gamma)$ is obtained from $\mathbf{H}^{3}$ by removing each closed hyperbolic half-space which intersects the sphere at infinity in a closed disk contained in $\Omega(\Gamma)$. The convex core $\mathcal{C}(N)$ of $N=\mathbf{H}^{3} / \Gamma$ is the quotient $\widetilde{\mathcal{C}}(\Gamma) / \Gamma$. Equivalently, the convex core of $N$ is the smallest convex submanifold of $N$ whose inclusion is a homotopy equivalence. (See Epstein and Marden [7] for further discussion of the convex core.)

The following proposition describes the basic relationship between the visual and convex cores of a hyperbolic 3-manifold $N$.

Proposition 2.2 Let $N=\mathbf{H}^{3} / \Gamma$ be a hyperbolic 3-manifold. Then, its visual core $\mathcal{V}(N)$ is contained in its convex core $\mathcal{C}(N)$. Moreover, the visual core is equal to the convex core if and only if the boundary $\partial \mathcal{C}(N)$ of the convex core is totally geodesic.

Proof of Proposition 2.2: For each point $x$ of $\mathbf{H}^{3}-\widetilde{\mathcal{C}}(\Gamma)$, there exists a hyperplane $H$ in $\mathbf{H}^{3}$ containing $x$ so that the circle at infinity $C$ of $H$ bounds a closed disk contained entirely in a component $\Delta$ of $\Omega(\Gamma)$. Thus, $h_{\Delta}(x)>\frac{1}{2}$, which implies that $x \notin \tilde{\mathcal{V}}(\Gamma)$. Therefore, $\widetilde{\mathcal{V}}(\Gamma) \subset \widetilde{\mathcal{C}}(\Gamma)$, which in turn implies that $\mathcal{V}(N) \subset \mathcal{C}(N)$

For each point $x$ of $\partial \widetilde{\mathcal{C}}(\Gamma)$, there exists a hyperplane $H$ in $\mathbf{H}^{3}$ containing $x$ so that the circle at infinity $C$ of $H$ bounds an open disk $D$ contained entirely in a component $\Delta$ of 
$\Omega(\Gamma)$. If $D$ does not equal $\Delta$, then $h_{\Delta}(x)>\frac{1}{2}$, which implies that $x \notin \widetilde{\mathcal{V}}(\Gamma)$. Therefore, $\widetilde{\mathcal{C}}(\Gamma)=\widetilde{\mathcal{V}}(\Gamma)$ if and only if each component of $\Omega(\Gamma)$ is a circular disc, which is equivalent to requiring that $\partial \mathcal{C}(N)$ be totally geodesic.

Proposition 2.2

\section{The visual core and coverings}

In this section, we develop a criterion, expressed in terms of limit sets, which guarantees that the visual core of a cover of a hyperbolic manifold embeds under the covering map. This criterion involves the introduction of two closely related notions of how a subgroup $\Gamma^{\prime}$ of a Kleinian group $\Gamma$ sits inside $\Gamma$.

We begin by observing that if $\Gamma^{\prime}$ is a precisely QF-embedded subgroup of a Kleinian group $\Gamma$, then the interior of $\mathcal{V}\left(N^{\prime}\right)$ embeds in $N$ under the covering map $\pi: N \rightarrow N^{\prime}$ (where $N=\mathbf{H}^{3} / \Gamma$ and $N^{\prime}=\mathbf{H}^{3} / \Gamma^{\prime}$ ). Here, a subgroup $\Gamma^{\prime}$ of a Kleinian group $\Gamma$ is precisely QF-embedded if, for each $\gamma \in \Gamma-\Gamma^{\prime}$, there is a component $\Delta$ of $\Omega\left(\Gamma^{\prime}\right)$ so that $\gamma\left(\Lambda\left(\Gamma^{\prime}\right)\right)$ is contained in $\bar{\Delta}, \operatorname{st}_{\Gamma^{\prime}}(\Delta)$ is quasifuchsian, and $\Delta$ is a Jordan domain.

Recall that a quasifuchsian group is a finitely generated Kleinian group whose limit set is a Jordan curve and which stabilizes both components of its domain of discontinuity. In particular, if $\Delta$ is a component of the domain of discontinuity $\Omega(\Gamma)$ of a Kleinian group $\Gamma$, $\operatorname{st}_{\Gamma^{\prime}}(\Delta)$ is quasifuchsian, and $\Delta$ is a Jordan domain, then $\Lambda\left(\operatorname{st}_{\Gamma^{\prime}}(\Delta)\right)=\partial \Delta$. If $\Gamma$ is finitely generated, then a component $\Delta$ of $\Omega(\Gamma)$ is a Jordan domain if and only if $\operatorname{st}_{\Gamma}(\Delta)$ is quasifuchsian, see Lemma 2 of Ahlfors [1] and Theorem 2 of Maskit [9]. Hence, a finitely generated subgroup $\Gamma^{\prime}$ of a Kleinian group $\Gamma$ is precisely QF-embedded if, for each $\gamma \in \Gamma-\Gamma^{\prime}$, there is a component $\Delta$ of $\Omega\left(\Gamma^{\prime}\right)$ so that $\gamma\left(\Lambda\left(\Gamma^{\prime}\right)\right)$ is contained in $\bar{\Delta}$ and $\Delta$ is a Jordan domain. Precisely QF-embedded subgroups arise naturally in Klein-Maskit combination theory, as we see in Section 5.

Proposition 3.1 Let $\Gamma$ be a Kleinian group and let $\Gamma^{\prime}$ be a precisely QF-embedded subgroup of $\Gamma$. Let $N=\mathbf{H}^{3} / \Gamma$ and $N^{\prime}=\mathbf{H}^{3} / \Gamma^{\prime}$, and let $\pi: N^{\prime} \rightarrow N$ be the covering map. Then, $\pi$ is an embedding restricted to the interior of the visual core $\mathcal{V}\left(N^{\prime}\right)$ of $N^{\prime}$.

Proof of Proposition 3.1: Since the interior of $\mathcal{V}\left(N^{\prime}\right)$ is an open submanifold (or possibly an open sub-orbifold, in the case that $\Gamma^{\prime}$ contains torsion) of $N^{\prime}$, it suffices to show that $\pi$ 
is injective on the interior of $\mathcal{V}\left(N^{\prime}\right)$. As $\mathcal{V}\left(N^{\prime}\right)$ is covered by $\tilde{\mathcal{V}}\left(\Gamma^{\prime}\right) \subset \mathbf{H}^{3}$, it suffices to show that if $\gamma \in \Gamma-\Gamma^{\prime}$, then $\gamma\left(\operatorname{int}\left(\widetilde{\mathcal{V}}\left(\Gamma^{\prime}\right)\right)\right) \cap \operatorname{int}\left(\widetilde{\mathcal{V}}\left(\Gamma^{\prime}\right)\right)$ is empty.

Let $\gamma$ be any element of $\Gamma-\Gamma^{\prime}$. Since $\Gamma^{\prime}$ is precisely QF-embedded in $\Gamma$, there exists a component $\Delta_{1}$ of $\Omega\left(\Gamma^{\prime}\right)$ so that $\gamma\left(\Lambda\left(\Gamma^{\prime}\right)\right) \subset \overline{\Delta_{1}}$, st ${ }_{\Gamma^{\prime}}\left(\Delta_{1}\right)$ is quasifuchsian, and $\Delta_{1}$ is a Jordan domain. Since $U=\widehat{\mathbf{C}}-\overline{\Delta_{1}}$ is a Jordan domain contained entirely in $\gamma\left(\Omega\left(\Gamma^{\prime}\right)\right)=\Omega\left(\gamma \Gamma^{\prime} \gamma^{-1}\right)$, there exists a component $\Delta_{1}^{\prime}$ of $\gamma\left(\Omega\left(\Gamma^{\prime}\right)\right)$ such that $U \subset \Delta_{1}^{\prime}$. In particular, $\overline{\Delta_{1}} \cup \Delta_{1}^{\prime}=\widehat{\mathbf{C}}$. Since $\partial \Delta_{1}$ is the limit set of the quasifuchsian group $\operatorname{st}_{\Gamma^{\prime}}\left(\Delta_{1}\right)$, it has measure zero, and so $h_{\Delta_{1}}(x)+h_{\Delta_{1}^{\prime}}(x) \geq 1$ for all $x \in \mathbf{H}^{3}$.

Since $h_{\Delta_{1}}$ is harmonic and non-constant, it cannot be locally constant. Thus, if $x$ is in $\operatorname{int}\left(\widetilde{\mathcal{V}}\left(\Gamma^{\prime}\right)\right)$, we see that $h_{\Delta_{1}}(x)<\frac{1}{2}$. Since $h_{\Delta_{1}^{\prime}}(x) \geq 1-h_{\Delta_{1}}(x)>\frac{1}{2}$, we see that $x$ does not lie in $\gamma\left(\operatorname{int}\left(\widetilde{\mathcal{V}}\left(\Gamma^{\prime}\right)\right)\right)$. Therefore $\gamma\left(\operatorname{int}\left(\widetilde{\mathcal{V}}\left(\Gamma^{\prime}\right)\right)\right) \cap \operatorname{int}\left(\widetilde{\mathcal{V}}\left(\Gamma^{\prime}\right)\right)$ is empty, as desired.

Proposition 3.1

A subgroup $\Gamma^{\prime}$ of a Kleinian group $\Gamma$ is nicely $Q F$-embedded if for each $\gamma \in \Gamma-\Gamma^{\prime}$, there exists a component $\Delta$ of $\Omega\left(\Gamma^{\prime}\right)$ such that $\gamma\left(\Lambda\left(\Gamma^{\prime}\right)\right)$ is contained in the closure of $\Delta$, st $\Gamma_{\Gamma^{\prime}}(\Delta)$ is quasifuchsian, $\Delta$ is a Jordan domain, and $\gamma\left(\Lambda\left(\Gamma^{\prime}\right)\right)$ does not contain $\partial \Delta$. (Notice that the only difference between the definition of precisely QF-embedded and nicely QF-embedded is the requirement that $\gamma\left(\Lambda\left(\Gamma^{\prime}\right)\right) \cap \partial \Delta \neq \partial \Delta$.) In particular, if $\Gamma^{\prime}$ is a finitely generated subgroup of a Kleinian group $\Gamma$, then $\Gamma^{\prime}$ is nicely QF-embedded if, for each $\gamma \in \Gamma-\Gamma^{\prime}$, there is a component $\Delta$ of $\Omega\left(\Gamma^{\prime}\right)$ so that $\gamma\left(\Lambda\left(\Gamma^{\prime}\right)\right)$ is contained in $\bar{\Delta}, \gamma\left(\Lambda\left(\Gamma^{\prime}\right)\right) \cap \partial \Delta \neq \partial \Delta$, and $\Delta$ is a Jordan domain. Nicely embedded QF-subgroups occur naturally in the study of algebraic and geometric limits, as we see in Section 4.

We next observe that if $\Gamma^{\prime}$ is nicely QF-embedded in $\Gamma$, then the visual core of $N^{\prime}=\mathbf{H}^{3} / \Gamma^{\prime}$ embeds in $N=\mathbf{H}^{3} / \Gamma$. Recall that if $\Gamma^{\prime}$ is just precisely QF-embedded then we only know that the interior of the visual core embeds. In Remark 2 at the end of Section 5, we note that summands of simple type II Klein-Maskit decompositions are precisely QF-embedded, but not nicely QF-embedded.

Proposition 3.2 Let $\Gamma$ be a Kleinian group and let $\Gamma^{\prime}$ be a finitely generated, nicely QFembedded subgroup of $\Gamma$. Let $N=\mathbf{H}^{3} / \Gamma$ and $N^{\prime}=\mathbf{H}^{3} / \Gamma^{\prime}$, and let $\pi: N^{\prime} \rightarrow N$ be the covering map. Then, $\pi$ is an embedding restricted to the visual core $\mathcal{V}\left(N^{\prime}\right)$ of $N^{\prime}$.

Proof of Proposition 3.2: We argue much as in the proof of Proposition 3.1 to show that $\pi$ is injective on $\mathcal{V}(N)$. Let $\gamma$ be any element of $\Gamma-\Gamma^{\prime}$. Since $\Gamma^{\prime}$ is nicely QF-embedded in 
$\Gamma$, there exists a component $\Delta_{1}$ of $\Omega\left(\Gamma^{\prime}\right)$ which is a Jordan domain, so that $\gamma\left(\Lambda\left(\Gamma^{\prime}\right)\right) \subset \overline{\Delta_{1}}$ and $\partial \Delta_{1}-\gamma\left(\Lambda\left(\Gamma^{\prime}\right)\right)$ is non-empty. Thus $\widehat{\mathbf{C}}-\overline{\Delta_{1}}$ is a proper subset of some component $\Delta_{1}^{\prime}$ of $\gamma\left(\Omega\left(\Gamma^{\prime}\right)\right)$. In particular, $\overline{\Delta_{1}} \cup \Delta_{1}^{\prime}=\widehat{\mathbf{C}}$ and $\Delta_{1} \cap \Delta_{1}^{\prime} \neq \emptyset$. Since $\partial \Delta_{1}$ is the limit set of a quasifuchsian group, it has measure zero. Thus, $h_{\Delta_{1}}(x)+h_{\Delta_{1}^{\prime}}(x)>1$ for all $x \in \mathbf{H}^{3}$.

If $x \in \tilde{\mathcal{V}}\left(\Gamma^{\prime}\right)$, then $h_{\Delta_{1}}(x) \leq \frac{1}{2}$. So, we see that $h_{\Delta_{1}^{\prime}}(x)>1-h_{\Delta_{1}}(x) \geq \frac{1}{2}$, which implies that $x$ does not lie in $\gamma\left(\widetilde{\mathcal{V}}\left(\Gamma^{\prime}\right)\right)$. Thus, $\gamma\left(\widetilde{\mathcal{V}}\left(\Gamma^{\prime}\right)\right) \cap \widetilde{\mathcal{V}}\left(\Gamma^{\prime}\right)$ is empty, which proves that $\pi$ is injective on the visual core $\mathcal{V}\left(N^{\prime}\right)$.

To verify that $\pi$ is an embedding restricted to $\mathcal{V}\left(N^{\prime}\right)$, it only remains to check that $\pi$ is proper. If not, then there must exist a sequence $\left\{x_{j}\right\}$ of points in $\mathcal{V}\left(N^{\prime}\right)$ which exits every compact subset of $N^{\prime}$, but such that $\left\{\pi\left(x_{j}\right)\right\}$ converges to a point $x$ in $N$. By passing to a subsequence, we may assume that $d\left(x_{j}, x_{j+1}\right) \geq 1$ and $d\left(\pi\left(x_{j}\right), x\right) \leq \frac{1}{3 j}$ for all $j$.

Let $\left\{\tilde{x}_{j}\right\}$ be a sequence of lifts of $\left\{x_{j}\right\}$ to $\mathbf{H}^{3}$. Since $d\left(\pi\left(x_{j}\right), \pi\left(x_{j+1}\right)\right)<\frac{1}{j}$ and $d\left(x_{j}, x_{j+1}\right) \geq$ 1 , for each $j$ there exists an element $\gamma_{j} \in \Gamma-\Gamma^{\prime}$ such that $d\left(\widetilde{x}_{j}, \gamma_{j}\left(\widetilde{x}_{j+1}\right)\right)<\frac{1}{j}$. Since $\Gamma^{\prime}$ is nicely QF-embedded, there exists, for each $j$, a component $\Delta_{j}$ of $\Omega\left(\Gamma^{\prime}\right)$ which is a Jordan domain whose closure contains $\gamma_{j}\left(\Lambda\left(\Gamma^{\prime}\right)\right)$. Since $\widetilde{x}_{j+1} \in \widetilde{\mathcal{V}}(\Gamma), \gamma_{j}(\widetilde{\mathcal{V}}(\Gamma)) \cap \widetilde{\mathcal{V}}(\Gamma)=\emptyset$, and $\gamma_{j}(\Lambda(\Gamma)) \subset \Delta_{j}$, we see that $h_{\Delta_{j}}\left(\gamma_{j}\left(\widetilde{x}_{j+1}\right)\right)>\frac{1}{2}$. Since $\widetilde{x}_{j} \in \widetilde{\mathcal{V}}\left(\Gamma^{\prime}\right), h_{\Delta_{j}}\left(\widetilde{x}_{j}\right) \leq \frac{1}{2}$. So, by continuity, there exists a point $\widetilde{q}_{j}$ between $\widetilde{x}_{j}$ and $\gamma_{j}\left(\widetilde{x}_{j+1}\right)$ such that $d\left(\widetilde{q}_{j}, \widetilde{x}_{j}\right)<\frac{1}{j}$ and $h_{\Delta_{j}}\left(\widetilde{q}_{j}\right)=\frac{1}{2}$. Thus, if $p^{\prime}: \mathbf{H}^{3} \rightarrow N^{\prime}$ is the obvious covering map and $q_{j}=p^{\prime}\left(\widetilde{q}_{j}\right)$, then $\left\{q_{j}\right\}$ is a sequence of points in $\partial \mathcal{V}\left(N^{\prime}\right)$ which exits every compact subset of $N^{\prime}$ such that $\left\{\pi\left(q_{j}\right)\right\}$ converges to a point $x$ in $N$.

Since $\Gamma^{\prime}$ is finitely generated, there exist only finitely many inequivalent components of $\Omega\left(\Gamma^{\prime}\right)$, so we may assume (by choosing different lifts of $q_{j}$, passing to a subsequence, and relabelling) that there exists a fixed component $\Delta_{0}$ of $\Omega\left(\Gamma^{\prime}\right)$ which is a Jordan domain, so that $h_{\Delta_{0}}\left(\tilde{q}_{j}\right)=\frac{1}{2}$ for all $j$. Let $\Gamma_{0}=\operatorname{st}_{\Gamma^{\prime}}\left(\Delta_{0}\right), N_{0}=\mathbf{H}^{3} / \Gamma_{0}$ and $p_{0}: \mathbf{H}^{3} \rightarrow N_{0}$ be the covering map. Since $h_{\Delta_{0}}\left(\tilde{q}_{j}\right)=\frac{1}{2}$, we conclude that $\tilde{q}_{j} \in \tilde{\mathcal{V}}\left(\Gamma_{0}\right)$. Let $y_{j}=p_{0}\left(\tilde{q}_{j}\right)$. Since $\left\{q_{j}\right\}$ exits every compact subset of $N^{\prime},\left\{y_{j}\right\}$ must exit every compact subset of $N_{0}$. Proposition 2.2 guarantees that the sequence $\left\{y_{j}\right\}$ lies entirely in the convex core $C\left(N_{0}\right)$ of $N_{0}$. Since $\Gamma^{\prime}$ is finitely generated and $\Delta_{0}$ is a Jordan domain, $\Gamma_{0}$ is quasifuchsian. Therefore, the $\epsilon$-thick part of the convex core,

$$
C\left(N_{0}\right)_{\epsilon}=\left\{y \in C\left(N_{0}\right) \mid \operatorname{inj}_{N_{0}}(y) \geq \epsilon\right\}
$$

is compact for all $\epsilon>0$, see Bowditch [5]. (Here, $\operatorname{inj}_{N_{0}}(y)$ denotes the injectivity radius of

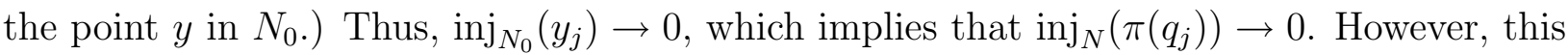


contradicts the fact that $\left\{\pi\left(q_{j}\right)\right\}$ converges in $N$.

Proposition 3.2

Remarks: (1) One may think of Proposition 3.1 as an analogue of Proposition 6.1 of [3], which asserts that if $\Gamma^{\prime}$ is a finitely generated, torsion-free, precisely embedded generalized web subgroup of $\Gamma$, then there is a compact core for $N^{\prime}$ which embeds (via the covering map $\pi: N \rightarrow N^{\prime}$ ) in $N$. That result may be generalized, using the same techniques as in [3], to show that if $\Gamma^{\prime}$ is a finitely generated, torsion-free precisely QF-embedded subgroup of $\Gamma$, then there is a compact core for $N^{\prime}$ which embeds (via the covering map $\pi$ ) in $N$. This generalization is the more direct topological analogue of Proposition 3.1.

(2) The arguments in the proofs of Propositions 3.1 and 3.2 may be used to show that larger subsets embed. Let $\widetilde{\mathcal{W}}\left(\Gamma^{\prime}\right)$ be the set of points $x \in \mathbf{H}^{3}$ such that $h_{\Delta}(x) \leq \frac{1}{2}$ for every component $\Delta$ of $\Omega\left(\Gamma^{\prime}\right)$ such that $\operatorname{st}_{\Gamma^{\prime}}(\Delta)$ is quasifuchsian, and set $\mathcal{W}\left(N^{\prime}\right)=\widetilde{\mathcal{W}}\left(\Gamma^{\prime}\right) / \Gamma^{\prime}$. Then one can adapt the proof of Proposition 3.1 to show that if $\Gamma^{\prime}$ is a precisely QF-embedded subgroup of $\Gamma$, then the interior of $\mathcal{W}\left(N^{\prime}\right)$ embeds in $N$. Similarly, one can adapt the proof of Proposition 3.2 to show that if $\Gamma^{\prime}$ is a finitely generated, nicely QF-embedded subgroup of $\Gamma$, then $\mathcal{W}\left(N^{\prime}\right)$ embeds in $N$.

(3) Note that the definitions for a precisely QF-embedded and of a nicely QF-embedded subgroup $\Gamma^{\prime}$ of a Kleinian group $\Gamma$ both make sense for an infinitely generated subgroup $\Gamma^{\prime}$. In fact, Proposition 3.1 as stated holds for infinitely generated, precisely QF-embedded subgroups. The reason that in the definitions we require both that the component $\Delta$ of $\Omega\left(\Gamma^{\prime}\right)$ be a Jordan domain and that $\operatorname{st}_{\Gamma^{\prime}}(\Delta)$ be quasifuchsian is that it is possible, by taking the Klein combination of a quasifuchsian group with an infinitely generated Kleinian group with trivial component subgroups, to construct an infinitely generated Kleinian group whose component subgroups are all quasifuchsian but the components of whose domain of discontinuity are not all simply connected, and so in particular cannot all be Jordan domains.

\section{Algebraic and geometric limits}

In an earlier paper [3], we proved that if the algebraic limit of a sequence of isomorphic Kleinian groups is a generalized web group, then it is a nicely QF-embedded subgroup of the geometric limit. In that paper, we used this result to establish that there is a compact core for the algebraic limit manifold which embeds in the geometric limit manifold, thus obtaining 
"topological" information about how the algebraic limit sits inside the geometric limit. In this section, we use the results of the previous section to obtain "geometric" information about how the algebraic limit sits inside the geometric limit.

We briefly recall the basic definitions from the theory of algebraic and geometric limits. We refer the interested reader to Jørgensen and Marden [8] for more details. Given a finitely generated group $G$, let $\mathcal{D}(G)$ denote the space of discrete, faithful representations of $G$ into $\mathrm{PSL}_{2}(\mathbf{C})$. A sequence $\left\{\rho_{i}\right\}$ in $\mathcal{D}(G)$ converges algebraically to $\rho$ if $\left\{\rho_{i}(g)\right\}$ converges to $\rho(g)$ for each $g \in G$.

A sequence $\left\{\Gamma_{j}\right\}$ of Kleinian groups converges geometrically to a Kleinian group $\widehat{\Gamma}$ if every element of $\widehat{\Gamma}$ is the limit of a sequence $\left\{\gamma_{j} \in \Gamma_{j}\right\}$ and if every accumulation point of every sequence $\left\{\gamma_{j} \in \Gamma_{j}\right\}$ lies in $\widehat{\Gamma}$. If $G$ is not virtually abelian and if $\left\{\rho_{i}\right\}$ converges to $\rho$ in $\mathcal{D}(G)$, then there is a subsequence $\left\{\rho_{j}(G)\right\}$ of $\left\{\rho_{i}(G)\right\}$ which converges geometrically to a Kleinian group $\widehat{\Gamma}$ which contains $\rho(G)$.

In this note, we restrict ourselves to sequences $\left\{\rho_{n}\right\}$ in $\mathcal{D}(G)$ so that $\left\{\rho_{n}\right\}$ converges algebraically to some $\rho \in \mathcal{D}(G)$ and so that $\left\{\rho_{n}(G)\right\}$ converges geometrically to $\widehat{\Gamma}$. The Kleinian group $\rho(G)$ is the algebraic limit of $\left\{\rho_{n}\right\}$, and $\widehat{\Gamma}$ is the geometric limit of $\left\{\rho_{n}(G)\right\}$. If $G$ is torsion-free, we refer to $\mathbf{H}^{3} / \rho(G)$ as the algebraic limit manifold and to $\mathbf{H}^{3} / \widehat{\Gamma}$ as the geometric limit manifold. Since $\rho(G) \subset \widehat{\Gamma}$, there is a natural covering map $\pi: \mathbf{H}^{3} / \rho(\Gamma) \rightarrow$ $\mathbf{H}^{3} / \widehat{\Gamma}$. In order to understand the relationship between the algebraic and geometric limits, it is important to understand how $\rho(G)$ "sits inside" $\widehat{\Gamma}$, which is closely related to understanding the covering map $\pi$.

A finitely generated Kleinian group $\Gamma$ is called a generalized web group if $\Omega(\Gamma)$ is nonempty and if every component subgroup of $\Gamma$ is quasifuchsian (or equivalently, if every component of $\Omega(\Gamma)$ is a Jordan domain). Theorem A from [3] asserts that if the algebraic limit is a generalized web group, then it is a nicely QF-embedded subgroup of the geometric limit.

Theorem 4.1 (Theorem A of [3]) Let $G$ be a finitely generated, torsion-free, non-abelian group, let $\left\{\rho_{j}\right\}$ be a sequence in $\mathcal{D}(G)$ converging algebraically to $\rho \in \mathcal{D}(G)$, and suppose that $\left\{\rho_{j}(G)\right\}$ converges geometrically to $\widehat{\Gamma}$. If $\rho(G)$ is a generalized web group, then $\rho(G)$ is a nicely $Q F$-embedded subgroup of $\widehat{\Gamma}$.

One may combine Theorem 4.1 with Proposition 3.2 to obtain "geometric" information about how the algebraic limit sits within the geometric limit in this case. 
Theorem 4.2 Let $G$ be a finitely generated, torsion-free, non-abelian group, let $\left\{\rho_{j}\right\} \subset$ $\mathcal{D}(G)$ be a sequence converging algebraically to $\rho \in \mathcal{D}(G)$, and suppose that $\left\{\rho_{j}(G)\right\}$ converges geometrically to $\widehat{\Gamma}$. If $\rho(G)$ is a generalized web group, then the visual core of $N=\mathbf{H}^{3} / \rho(G)$ embeds in $\widehat{N}=\mathbf{H}^{3} / \widehat{\Gamma}$ under the covering map $\pi: N \rightarrow \widehat{N}$.

Remarks: (1) One may think of Theorem 4.2 as one way to generalize Proposition 3.2 from [4], which shows that if the algebraic limit is a maximal cusp, then the convex core of the algebraic limit manifold embeds in the geometric limit manifold under the covering map. In fact, one may view our Theorem 4.2 and the result from [3] that asserts that, under the same assumptions, a compact core for the algebraic limit manifold embeds in the geometric limit manifold, as two different generalizations of Proposition 3.2 from [4].

(2) In general, even if the algebraic limit is a generalized web group, the convex core of the algebraic limit manifold need not embed in the geometric limit manifold.

(3) The examples given in [2] illustrate the point that the visual core of the algebraic limit manifold need not embed in the geometric limit manifold in the case that the algebraic limit is not a generalized web group.

\section{$5 \quad$ Klein-Maskit Combination}

In this section, we discuss the relationship between the visual core and the operation of Klein-Maskit combination. We restrict our entire discussion to Klein-Maskit combination along component subgroups. For a more complete discussion of Klein-Maskit combination see Maskit [10]. In this setting, we see that the interior of the visual core of a summand of a Klein-Maskit decomposition of a hyperbolic 3-manifold embeds in the manifold.

There are two types of Klein-Maskit combination. The first, type I, corresponds topologically to gluing 2 hyperbolic 3-manifolds together along incompressible components of their conformal boundary. The second, type II, corresponds topologically to gluing together two incompressible components of the conformal boundary of a single hyperbolic 3-manifold.

The following theorem summarizes the relevant properties of Klein-Maskit combination of type I along a component subgroup (see Theorem VII.C.2 in [10]).

Theorem 5.1 (Klein-Maskit combination I) Let $\Gamma_{1}$ and $\Gamma_{2}$ be Kleinian groups, and let $\Phi=\Gamma_{1} \cap \Gamma_{2}$. Suppose that $\Phi$ is a quasifuchsian group which is a component subgroup of both $\Gamma_{1}$ and $\Gamma_{2}$, and that $\Lambda\left(\Gamma_{1}\right)$ and $\Lambda\left(\Gamma_{2}\right)$ lie in the closures of different components of $\Omega(\Phi)$. Then, 
1. $\Gamma=\left\langle\Gamma_{1}, \Gamma_{2}\right\rangle$ is a Kleinian group isomorphic to the amalgamated free product of $\Gamma_{1}$ and $\Gamma_{2}$ along $\Phi$;

2. $\Gamma_{1}$ and $\Gamma_{2}$ are nicely $Q F$-embedded subgroups of $\Gamma$;

3. If $\gamma \in \Gamma-\Gamma_{i}$, then $\gamma\left(\Lambda\left(\Gamma_{i}\right)\right)$ is contained in a component $\Delta$ of $\Omega\left(\Gamma_{i}\right)$ which is $\Gamma_{i^{-}}$ equivalent to the component $\Delta_{i}$ of $\Omega\left(\Gamma_{i}\right)$ bounded by $\Lambda(\Phi)$. Moreover, $\partial \Delta-\gamma\left(\Lambda\left(\Gamma_{i}\right)\right)$ is non-empty; and

4. $\mathbf{H}^{3} / \Gamma$ is homeomorphic to the manifold (or orbifold) obtained from $\left(\mathbf{H}^{3} \cup \Delta_{1}\right) / \Gamma_{1}$ and $\left(\mathbf{H}^{3} \cup \Delta_{2}\right) / \Gamma_{2}$ by identifying $\Delta_{1} / \Phi$ with $\Delta_{2} / \Phi$.

In this case, we say that $\Gamma_{1}$ is a summand of a simple type I Klein-Maskit decomposition of $\Gamma$. Combining property (2) of Theorem 5.1 with Proposition 3.2 yields the following result:

Proposition 5.2 Let $\Gamma_{1}$ be a finitely generated Kleinian group which is a summand of a simple type I Klein-Maskit decomposition of $\Gamma$, and set $N_{1}=\mathbf{H}^{3} / \Gamma_{1}$ and $N=\mathbf{H}^{3} / \Gamma$. Then, the visual core $\mathcal{V}\left(N_{1}\right)$ of $N_{1}$ embeds in $N$ (via the covering map $\pi: N_{1} \rightarrow N$ ).

If $\Gamma_{1}$ is not finitely generated, the techniques in the proof of Proposition 3.2 may be adapted to show that $\mathcal{V}\left(N_{1}\right)$ still embeds in $N$. More simply, one may combine property (2) of Theorem 5.1 with Proposition 3.1 to obtain the following weaker result:

Proposition 5.3 Let $\Gamma_{1}$ be a summand of a simple type I Klein-Maskit decomposition of a Kleinian group $\Gamma$, and set $N_{1}=\mathbf{H}^{3} / \Gamma_{1}$ and $N=\mathbf{H}^{3} / \Gamma$. Then, the interior of the visual core $\mathcal{V}\left(N_{1}\right)$ of $N_{1}$ embeds in $N$ (via the covering map $\pi: N_{1} \rightarrow N$ ).

Moreover, if $\Gamma$ is a simple type I Klein-Maskit combination of $\Gamma_{1}$ and $\Gamma_{2}$, we may find a larger subset of $N_{1}$ which embeds in $N$. Consider the sets

$$
\widetilde{\mathcal{X}_{i}}=\left\{x \in \mathbf{H}^{3} \mid h_{\Delta}(x) \leq \frac{1}{2} \text { for all components } \Delta \text { of } \Omega\left(\Gamma^{\prime}\right) \text { equivalent in } \Gamma_{i} \text { to } \Delta_{i}\right\} .
$$

Clearly, the visual hull $\tilde{\mathcal{V}}\left(\Gamma_{i}\right)$ of $\Gamma_{i}$ is contained in $\widetilde{\mathcal{X}_{i}}$. Let $\mathcal{X}_{i}=\widetilde{\mathcal{X}_{i}} / \Gamma_{i} \subset N_{i}=\mathbf{H}^{3} / \Gamma_{i}$. Condition (3) above and the arguments in the proof of Proposition 3.2 can then be used to show that $\mathcal{X}_{i}$ embeds in $N=\mathbf{H}^{3} / \Gamma$.

The following theorem summarizes the relevant properties of Klein-Maskit combination of type II along a component subgroup (see Theorem VII.E.5 in [10]). 
Theorem 5.4 (Klein-Maskit combination II) Let $\Gamma_{1}$ be a Kleinian group, and let $\Delta$ and $\Delta^{\prime}$ be components of $\Omega\left(\Gamma_{1}\right)$ which are Jordan domains. Suppose that $\Phi=\operatorname{st}_{\Gamma_{1}}(\Delta)$ and $\Phi^{\prime}=\operatorname{st}_{\Gamma_{1}}\left(\Delta^{\prime}\right)$ are quasifuchsian and are not conjugate by an element of $\Gamma_{1}$. Let $\gamma$ be a Möbius transformation which conjugates $\Phi^{\prime}$ to $\Phi$, and assume that $\gamma\left(\Lambda\left(\Gamma_{1}\right)\right)$ and $\Lambda\left(\Gamma_{1}\right)$ lie in the closures of different components of $\Omega(\Phi)$. Then,

1. $\Gamma=\left\langle\Gamma_{1}, \gamma\right\rangle$ is a Kleinian group isomorphic to the HNN-extension of $\Gamma_{1}$ with stable letter $\gamma$ and associated subgroups $\Phi$ and $\Phi^{\prime}$;

2. $\Gamma_{1}$ is a precisely $Q F$-embedded subgroup of $\Gamma$;

3. If $\gamma \in \Gamma-\Gamma_{1}$, then $\gamma\left(\Lambda\left(\Gamma_{1}\right)\right)$ is contained in a component of $\Omega\left(\Gamma_{1}\right)$ which is $\Gamma_{1}$-equivalent to either $\Delta$ or $\Delta^{\prime}$; and

4. $\mathbf{H}^{3} / \Gamma$ is homeomorphic to the manifold (or orbifold) obtained from $\left(\mathbf{H}^{3} \cup \Delta \cup \Delta^{\prime}\right) / \Gamma_{1}$ by identifying $\Delta / \Phi$ with $\Delta^{\prime} / \Phi^{\prime}$ by the homeomorphism determined by $\gamma$.

In this case, we say that $\Gamma_{1}$ is a summand of a simple type II Klein-Maskit decomposition of $\Gamma$. Combining property (2) of Theorem 5.4 with Proposition 3.1 yields the following result:

Proposition 5.5 Let $\Gamma_{1}$ be a summand of simple type II Klein-Maskit decomposition of $\Gamma$, and set $N_{1}=\mathbf{H}^{3} / \Gamma_{1}$ and $N=\mathbf{H}^{3} / \Gamma$. Then, the interior of the visual core $\mathcal{V}\left(N_{1}\right)$ of $N_{1}$ embeds in $N$ (via the covering map $\pi: N_{1} \rightarrow N$ ).

As in the type I situation, if $\Gamma_{1}$ is a summand of simple type II Klein-Maskit decomposition of $\Gamma$, we may find a larger subset of $N_{1}$ which embeds in $N$. Consider the set

$$
\widetilde{\mathcal{X}}=\left\{x \in \mathbf{H}^{3} \mid h_{\Delta}(x) \leq \frac{1}{2} \text { for all components } \Delta \text { of } \Omega\left(\Gamma^{\prime}\right) \text { equivalent in } \Gamma_{1} \text { to } \Delta \text { or } \Delta^{\prime}\right\},
$$

which clearly contains the visual hull $\widetilde{\mathcal{V}}\left(\Gamma_{1}\right)$ of $\Gamma_{1}$. Let $\mathcal{X}=\widetilde{\mathcal{X}} / \Gamma_{1} \subset N_{1}$. Condition (3) above and the arguments in the proof of Proposition 3.1 can be used to show that the interior of $\mathcal{X}$ embeds in $N=\mathbf{H}^{3} / \Gamma$.

In general, if a Kleinian group $\Gamma$ can be built from $\Gamma_{1}$ and a collection of other Kleinian groups by repeatedly performing Klein-Maskit combinations of types I and/or II along component subgroups, we say that $\Gamma_{1}$ is a summand of a Klein-Maskit decomposition of $\Gamma$. By applying Propositions 5.3 and 5.5, we obtain the following summation of the results of this section. 
Theorem 5.6 Let $\Gamma_{1}$ be a summand of a Klein-Maskit decomposition of $\Gamma$. If $N_{1}=\mathbf{H}^{3} / \Gamma_{1}$ and $N=\mathbf{H}^{3} / \Gamma$, then the interior of the visual core $\mathcal{V}\left(N_{1}\right)$ of $N_{1}$ embeds in $N$ (via the covering map $\pi: N_{1} \rightarrow N$ ).

Remarks: (1) The definition of the visual core was suggested by Thurston's reproof of the Klein-Maskit combination theorems. In our notation, Thurston shows that in the type I decomposition, $N=\mathbf{H}^{3} / \Gamma$ is obtained from $\mathcal{X}_{1}$ and $\mathcal{X}_{2}$ by identifying points in their boundaries. (In general, one must be a little careful since $\mathcal{X}_{i}$ need not be a submanifold.) Similarly, in the type II situation he shows that $N$ is obtained from $\mathcal{X}$ by identifying points in the boundary. (This proof is discussed in outline in Section 8 of Morgan [11].)

(2) Notice that if $\Gamma_{1}$ is a summand of a simple type II Klein-Maskit decomposition of a Kleinian group $\Gamma$, then $\Gamma_{1}$ is a precisely QF-embedded subgroup of $\Gamma$, but is not a nicely QF-embedded subgroup. In this same case, the interior of the visual cover of $N_{1}$ embeds in $N$, but the visual core itself does not.

(3) Corollary D of [3] asserts that if the algebraic limit of a sequence of isomorphic Kleinian groups is a generalized web group, then it is a summand of a Klein-Maskit decomposition of the geometric limit. Hence, there is a close relationship between Theorems 4.2 and 5.6.

\section{References}

[1] L. V. Ahlfors, "The structure of a finitely generated Kleinian group," Acta Math. 122 (1969), 1-17.

[2] J. W. Anderson and R. D. Canary, "Algebraic limits of Kleinian groups which rearrange the pages of a book," Invent. Math., 126 (1996), 205-214.

[3] J. W. Anderson and R. D. Canary, "Cores of hyperbolic 3-manifolds and limits of Kleinian groups," Amer. J. Math. 118 (1996), 745-779.

[4] J. W. Anderson, R. D. Canary, M. Culler, and P. Shalen, "Free Kleinian groups and volumes of hyperbolic 3-manifolds," J. Diff. Geom. 44 (1996), 738-782.

[5] B.H. Bowditch, "Geometrical finiteness for hyperbolic groups," J. Func. Anal. 113(1993), 245-317.

[6] R. D. Canary, "Ends of hyperbolic 3-manifolds," J.A.M.S. 6 (1993), 1-35.

[7] D. B. A. Epstein and A. Marden, "Convex hulls in hyperbolic spaces, a theorem of Sullivan, and measured pleated surfaces," in Analytical and Geometrical Aspects of Hyperbolic Spaces, Cambridge University Press, 1987, 113-253. 
[8] T. Jørgensen and A. Marden, "Algebraic and geometric convergence of Kleinian groups," Math. Scand. 66 (1990), 47-72.

[9] B. Maskit, "On boundaries of Teichmüller spaces and on Kleinian groups II," Ann. of Math. 91 (1970), 607-639.

[10] B. Maskit, Kleinian groups, Springer-Verlag, 1988.

[11] J.W. Morgan, "On Thurston's uniformization theorem for three-dimensional manifolds," in The Smith Conjecture, ed. by J. Morgan and H. Bass, Academic Press, 1984, 37-125.

[12] T. Soma, "Function groups in Kleinian groups," Math. Annalen 292 (1992), 181-190. 